\title{
Allergic contact dermatitis from pyridine in Karl Fischer reagent
}

\author{
C. Knegt-Junk, L. Geursen-Reitsma and T. van Joost \\ Department of Dermatology and Venereology, Academic Hospital Rotterdam Dijkzigt, Dr. Molewaterplein 40, \\ 3015 GD Rotterdam, the Netherlands
}

Key words: allergic contact dermatitis; pyridine; CAS 110-86-1; pyrimidine; Karl Fischer reagent; laboratory worker; occupational.

A 36-year-old laboratory technician developed eczema on the fingertips and interdigital spaces of both hands after working for 6 months in a chemical laboratory. At first, it improved at the weekends, but later it became more persistent. Dermatitis spread to both sides of her hands. Wearing gloves did not improve the symptoms.

She was patch tested with the European standard series (Hermal-Trolab, Germany). Positive reactions were observed after 2 and 3 days to the following: cobalt chloride $1 \%$ pet. $++1++$, nickel sulfate $5 \%$ pet. $++1+++$, fragrance $\operatorname{mix} 8 \%$ pet. $-1+++$ and Kathon CG $0.01 \%$ a.i. aq. $-1++$.

In spite of strict avoidance of these allergens, there was still gradual worsening of her hand dermatitis. She was therefore tested with various occupational contactants, including soap and detergents for cleaning

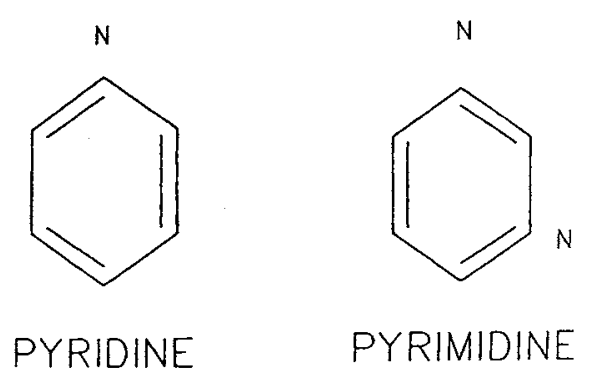

Fig. I. Chemical structures of pyridine and pyrimidine. glassware. The only positive reaction was observed with Karl Fischer reagent* containing pyridine mix. This was negative after 2 days but strongly positive after 3 days $(+++)$. Pyridine mix was used by the patient, in the assessment of chemicals for water quality, in a closed system because of its toxicity (1). The patient refused a patch test with a dilution of pyridine because of this toxicity. Besides pyridine, Karl Fischer reagent contains iodine and sulfur dioxide. No patch test reactions to these 2 suspect chemicals were seen.

Therefore, pyridine itself was most probably responsible for the allergic reactions. To our knowledge, crosssensitization between pyridine and pyrimidine (Fig. 1), which is present in some barbiturates (Thiopental), has not been described. Sensitization and clinical allergy apparently occurred due to leakage of material, which could not be avoided in spite of wearing gloves. As a consequence, this patient decided not to continue her laboratory work.

\section{Reference}

1. Windholz M, Budavari S, Blumetti R, Otterbein E. The Merck Index, eds. Merck \& Co., Inc. Rehway, NJ, USA, 1983: 7869-7870.

* Not to be confused with Fischer's reagent, used as a test for sugars, which contains sodium acetate and phenylhydrazine hydrochloride.

\section{Allergic contact dermatitis from multiple sources of MCI/MI biocide and formaldehyde in a printer}

\author{
C. M. REID AND R. J. G. RYCROFT \\ St John's Institute of Dermatology, St Thomas's Hospital, London SE1 7EH, UK
}

Key words: occupational; allergic contact dermatitis; airborne contact dermatitis; printing industry; 5-chloro-2methyl-4-isothiazolin-3-one; 2-methyl-4-isothiazolin-3-one; MCI/MI; formaldehyde; biocides; fountain solutions; gum arabic; skin care products.

\section{Case Report}

A 48-year-old printer presented with a 12-month history of vesicular palmar eczema, more recently spreading intermittently to his eyelids. There was no personal history of atopy but his sister had asthma and eczema.

Patch testing based on the European standard series showed positive reactions to formaldehyde $1 \%$ aq.
$(++)$, quaternium-15 (a formaldehyde-releaser) $1 \%$ aq. $(+), \mathrm{MCI} / \mathrm{MI}$ (5-chloro-2-methyl-4-isothiazolin-3-one/ 2-methyl-4-isothiazolin-3-one) $100 \mathrm{ppm}$ a.i. aq. $(++)$, and his barrier cream $(+)$ at 2 and 4 days.

Information from their manufacturers showed that his hand cleanser contained MCI/MI and that formaldehyde was present in his barrier cream. All 3 fountain 